\title{
Contributors to the 2014 IMIA Yearbook of Medical Informatics
}

Editors:

Marie-Christine Jaulent

INSERM-LIMISS UMRSI142

Paris, France

marie-christine.jaulent@crc.jussieu.fr

Christoph U. Lehmann

Vanderbilt University

Departments of Pediatrics and Biomedical Informatics

Nashille, TN, USA

christoph.u.lehmann@Vanderbilt.Edu

Brigitte Séroussi

UPMC Univ Paris 06 \& UMR S 1142, LIMICS

Tenon Hospital, AP-HP

Paris, France

brigitte.seroussi@tnn.aphp.fr

\section{Editorial Assistant:}

Martina Hutter

Heidelberg University Hospital- ZIM

Heidelberg, Germany

yearbook@imia-services.org

\section{Advisory Board:}

Reinhold Haux

Peter L. Reichertz Institute for Medical Informatics

University of Braunschweig - Institute of Technology and

Hannover Medical School

Braunschweig, Germany

reinhold.haux@plri.de

Victor Maojo

Universidad Politécnica de Madrid

Departamento de Inteligencia Aritificial

Facultad de Informatica

Madrid, Spain

vmaojo@fi.upm.es

George Mihalas

University of Medicine and Pharmacy

Dept. of Medical Informatics

Timisoara, Romania

mihalas@EFMl.nfo

\section{Section Editors:}

Xavier Aimé

INSERM

Paris, France

xavier.aime@inserm.fr

Jacques Bouaud

AP-HP \& LIMICS, UMRS 1142

Paris, France

jacques.bouaud@sap.aphp.fr

Jean Charlet

AP-HP \& LIMICS, UMRS 1142

Paris, France

jean.charlet@upmc.fr

Rémy Choquet

AP-HP National Database for Rare Diseases \& INSERM

Paris, France

remy.choquet@nck.ophp.fr
Emmanuel Conchon

IRIT-IRT \& ISIS

Castres, France

emmanuel.conchon@irit.fr

Marc Cuggia

DIM - CHU Pontchaillou

Rennes, France

marc.cuggia@univ-rennesl.fr

Christel Daniel

INSERM

CCS Patient - AP-HP

Paris, France

christel.daniel@crc.jussieu.fr

Stefan J. Darmoni

Roven University Hospital, TIBS, LITIS EA 4108

Department of BioMedical Informatics

Roven, France

stefan.darmoni@chu-roven.fr

Gunnar Declerck

INSERM UMRS 1142 (LIMICS), Sorbonne Universités

Univ. Paris 06 (UPMC), Sorbonne Paris Cité

Univ. Paris 13, COSTECH, Univ. de Technologie de Compiègne

Paris, France

gunnar.declerck@upmc.fr

Nassim Douali

INSERM-LIMICS UMRS1142

Paris, France

nassim.douali@crc.jussieu.fr

Nicolas Griffon

INSERM, U1 142, LIMICS \& Roven University Hospital

Paris, France

nicolas.griffon@chu-rouen.fr

Vassilis Koutkias

INSERM, U1142, LIMICS

Paris, France

E-mail: vasileios.koutkias@inserm.fr

Jean-Baptiste Lamy

University Paris 13, Sorbonne Paris Cité

\& UMR S 1142, LIMICS

jibalamy@free.fr

Thierry Lecroq

Normandie Univ., University of Rouen

Information Processing in Biology \& Health

Mont-Saint-Aignan, France

thierry.lecroq@univ-rouen.fr

Alexandre Moreau-Gaudry

CNRS - INSERM

Laboratoire TIMC-IMAG UMR 5525

UJF-Grenoble 1 - CIT803

Grenoble, France

alexandre.moreau-gaudry@imag.fr

Lina F. Soualmia

Normandie Univ., University of Rouen

Information Processing in Biology \& Health

Mont-Saint-Aignan, France

lina.soualmia@chu-rouen.fr
Nathalie Souf

IRIT-Elipse

Castres, France

nathalie.souf@irit.fr

Pascal Staccini

INSERM UMR 912

Université Nice-Sophia Antipolis

Nice, France

pascal.staccini@unice.fr

Frantz Thiessard

CHU de Bordeaux

Pole de sante publique

Bordeaux, France

frantz.thiessard@isped.u-bordeaux2.fr

Laurent Toubiana

INSERM UMRS 1142 LIMICS

Université Pierre et Marie Curie - Paris 6

Paris, France

laurent.toubiana@inserm.fr

Sandrine Voros

CNRS - INSERM

Laboratoire TIMC-IMAG UMR 5525

UJF-Grenoble 1

Grenoble, France

sandrine.voros@imag.fr

Regional Editors:

Karen Greenwood

AMIA

Bethesda, MD, USA

karen@amia.org

Don Newsham

COACH: Canada's Health Informatics Association Toronto, ON, Canada

dnewsham@coachorg.com

Alexander Hörbst

UMIT - Private University for Health Sciences,

Medical Informatics and Technology

Hall in Tirol, Austria

alexander.hoerbst@umit.at

Ghislain B. Kovematchoua Tchuitcheu

IMIA Vice President for HELINA

kovematchova@helina-online.org

Ying Wu

School of Nursing, Capital Medical University

Beiiing P.R.China

helenywapami@vip.163.com

Carola Hullin Lucay Cossio

IMIA LAC President

carolhullin@hotmail.com

IMIA Executive Director:

Peter J. Murray

Lincoln, Lincolnshire, UK

imia@imia-services.org 